\title{
The parallel postulate- return of the roaring lion
}

S.Kalimuthu

\author{
SF 211, Kanjampatti P.O, Pollachi Via,Tamil Nadu 642003, India
}

postulate.kalimuthu0@gmail.com

Abstract: In this work, without assuming the fifth Euclidean postulate, the author established the following theorems: 1.There exists a spherical quadrilateral whose interior angle sum is equal to 360 degrees. 2 .The sum of the interior angles of a triangle is a straight angle.

Keywords: Euclid, elements, postulates, non-Euclidean geometries, number theory, algebraic applications

MSC: 11A99, 08C99, 51M04

PACS: $02.40 . \mathrm{Ky}, 02.40 . \mathrm{Dr}$

Introduction

A brief history of evolution and revolution of geometry

The word geometry derives from the Greek, geo (earth) and metric (measure). Geometry is the study of shapes and configurations such as object points, lines and circles being the most basic of these. It attempts to understand and classify spaces in various mathematical contexts. For a space with lots of symmetries, the study naturally focuses on properties which are invariant (remaining the same) under the symmetries.

Geometry is perhaps is the most elementary of the sciences that enable man, by purely intellectual process, to make predictions (based on observations) about physical world. The power of geometry, in the sense of accuracy and utility of these deductions, is impressive and has been a motivation for the study of logic in geometry (Coxter, 1961, 1988; Sivasubramaniyan, 2009). The origins of geometry and number theory dates back 32000BC.

About 25000BC: Early geometric designs used.

About 5000BC: A decimal number system was in use in Egypt. About 4000BC: Babylonian and Egyptian calendars in use.

About 3400BC: The first symbols for numbers, simple straight lines, are used in Egypt.

About 3000BC: The abacus was developed in the Middle East and in areas around the Mediterranean. Hieroglyphic numerals in use in Egypt. Babylonians began to use a sexagesimal number system for recording financial transactions. It is a placevalue system without a zero place value.

About 2770BC: Egyptian calendar used.

About 2000BC: Harappans adopt a uniform decimal system of weights and measures.

About 1950BC: Babylonians solved quadratic equations.

About 1900BC: The Moscow papyrus (also called the Golenishev papyrus) was written. It gave details of Egyptian geometry

About 1850BC: Babylonians knew Pythagoras's Theorem.

About 1800BC: Babylonians used multiplication tables.

About 1750BC: The Babylonians solved linear and quadratic algebraic equations, compile tables of square and cube roots. They used Pythagoras's theorem and used mathematics to extend knowledge of astronomy.

About 1700BC: The Rhind papyrus (sometimes called the Ahmes papyrus) was written. It showed that Egyptian mathematics had developed many techniques to solve problems. Multiplication is based on repeated doubling, and division uses successive halving.
About 1400BC: About this date a decimal number system with no zero started to be used in China.

About 750BC: Manava wrote a Sulbasutra.

About 600BC: Apastamba wrote the most interesting Indian Sulbasutra from a mathematical point of view

575BC: Thales brought Babylonian mathematical knowledge to Greece. He used geometry to solve problems such as calculating the height of pyramids and the distance of ships from the shore.

530BC: Pythagoras of Samos moved to Croton in Italy and teaches mathematics, geometry, music, and reincarnation.

About 500BC: The Babylonian sexagesimal number system was used to record and predict the positions of the Sun, Moon and planets Panini's work on Sanskrit grammar is the forerunner of the modern formal language theoryln India, the vedic period findings in geometry (Rekha-Ganit) include construction of a square, construction of a square equal in area to a given rectangle, construction of a square equal in perimeter to a circle. Mathematicians of vedic period knew sutras related to construction of mounds for yagas and altars for keeping fire. These sutras indicated knowledge of the form $a+b=c$ which we call Pythagorean theorem. Thus we find that the Greek mathematician Pythagoras $(600 \mathrm{BC})$ was NOT the first to discover this theorem. Pythagoras was familiar with Upanishads and learnt his basic geometry from the Sulvasutras. History of Indian mathematics used to begin by describing the geometry contained in Sulvasutras. An early statement of what is commonly known as Pythagoras theorem is to be found in Baudhayana's (800 BC). The chord which is stretched across the diagram of a square produces an area of double the size. The earliest recorded beginnings of geometry can be traced to ancient Mesopotamia, Egypt, and the Indus Valley from around 3000 BC. Early geometry was a collection of empirically discovered principles concerning lengths, angles, areas, and volumes, which were developed to meet some practical need in surveying, construction, astronomy, and various crafts. The earliest known texts on geometry are the Egyptian Rhind Papyrus and Moscow Papyrus, the Babylonian clay tablets, and the Indian Shulba Sutras, while the Chinese had the work of Mozi, Zhang Heng, and the Nine Chapters on the Mathematical Art, edited by Liu Hui.

Euclid's The Elements of Geometry (c. 300 BC) was one of the most important early texts on geometry, in which he presented geometry in an ideal axiomatic form, which came to be known as Euclidean geometry. The treatise is not, as is sometimes thought, a compendium of all that Hellenistic mathematicians knew about geometry at that time; rather, it is an elementary introduction to it; Euclid himself wrote eight more advanced books on geometry. We know from other references that Euclid's was not the first elementary geometry textbook, but the others fell into disuse and were lost.

In the Middle Ages, Muslim mathematicians contributed to the development of geometry, especially algebraic geometry and geometric algebra. Al-Mahani (b. 853) conceived the idea of reducing geometrical problems such as duplicating the cube to problems in 
algebra. Thābit ibn Qurra (known as Thebit in Latin) (836901) dealt with arithmetical operations applied to ratios of geometrical quantities, and contributed to the development of analytic geometry. Omar Khayyám (1048-1131) found geometric solutions to cubic equations, and his extensive studies of the parallel postulate contributed to the development of NonEuclidian geometry.

In the early 17th century, there were two important developments in geometry. The first, and most important, was the creation of analytic geometry, or geometry with coordinates and equations, by René Descartes (15961650) and Pierre de Fermat (1601-1665). This was a necessary precursor to the development of calculus and a precise quantitative science of physics. The second geometric development of this period was the systematic study of projective geometry by Girard Desargues (15911661). Projective geometry is the study of geometry without measurement, just the study of how points align with each other.

Two developments in geometry in the nineteenth century changed the way it had been studied previously. These were the discovery of non-Euclidean geometries by Lobachevsky, Bolyai and Gauss and of the formulation of symmetry as the central consideration in the Erlangen Programme of Felix Klein (which generalized the Euclidean and non Euclidean geometries). Two of the master geometers of the time were Bernhard Riemann, working primarily with tools from mathematical analysis, and introducing the Riemann surface, and Henri Poincaré, the founder of algebraic topology and the geometric theory of dynamical systems, a sufficient supply. As a consequence of these major changes in the conception of geometry, the concept of "space" became something rich and varied, and the natural background for theories as different as complex analysis and classical mechanics. The traditional type of geometry was recognized as that of homogeneous spaces, those spaces which have of symmetry, so that from point to point they look just the same.

It was Euclid of Alexandria in Greece who wrote the first scientific text of geometry. He compiled all the available theorems and gave proof by introducing and assuming 23 definitions, 5 common notions and 5 postulates. His work is known as ELEMENTS.

Definitions (23)

1. A point is that which has no part.

2. A line is breathless length.

3. The ends of a line are points.

4. A straight line is a line which lies evenly with the points on itself.

5. A surface is that which has length and breadth only.

6 . The edges of a surface are lines.

7. A plane surface is a surface which lies evenly with the straight lines on itself.
8. A plane angle is the inclination to one another of two lines in a plane which meet one another and do not lie in a straight line.

9. And when the lines containing the angle are straight, the angle is called rectangle.

10. When a straight line standing on a straight line makes the adjacent angles equal to one another, each of the equal angle is right, and the straight line standing on the other is called a perpendicular to that on which it stands.

11. An obtuse angle is an angle greater than a right angle.

12. An acute angle is an angle less than a right angle.

13. A boundary is that which is an extremely of anything.

14. A figure is that which is contained by any boundary or boundaries.

15. A circle is a plane figure contained by one line such that all the straight line falling upon it from one point among those lying within the figure equal one another.

16. And the point is called the center of the circle.

17. A diameter of the circle is any straight line drawn to the center and terminated in both directions.

18. A semi circle is the figure contained by the diameter and the circumference cut off by it. And the center of the semi circle is the same as that of the circle.

19. Rectilinear figures are those which are contained by straight lines trilateral figures being those contained by three, quadrilateral those contained by four and multilateral those contained by more than four straight lines.

20. Oftrilateral figures, an equilateral triangle is that which has its three sides equal an isoscelestriangle that which has two of its sides are alone equal and a scalene triangle is that which has its three sides unequal.

21. Further, of trilateral figures, a right angles triangle is that which has a right angle, an obtuse angles triangle is that which has an obtuse angleand an acute angled triangle that which has its three angles acute.

22. Of quadrilateral figures, a square is that which is both equilatereal and right angled, an oblong that which is right angled but not equilateral, a rombhus that which is equilateral but not right angles, and a rhomboid that which has its opposite sides and angles equal to one another but is neither equilateral nor right angled. And let quadrilaterals other than these are called trapezia.

23. Parallel straight lines are straight lines which, being in the same plane, and being produced indefinitely in both directions, do not meet one another in either directions.

Postulates (5)

1. To draw a straight line from any point to any point.

2. To produce a finite straight line continuously in a straight line. 
3. To describe a circle with any center and radius.

4. That all right angles equal to one another.

5. If a straight line falling on two straight lines makes the interior angles on the same sideless than two right angles, if produced indefinitely meet on the side on which are the angles less than the two right angles.

Common Notions (5)

1. Things which equal the same thing also equal one another.

2. If the equals are added to the equals, then the wholes are equal.

3. If equals are subtracted from equals, then the remainders are equal.

4. Things which coincide with one another equal one another.

5 . The whole is greater than the part.

The first 28 propositions of Elements I

The following first 28 propositions of Elements do not use the parallel postulate

1. To construct an equilateral triangle on a given finite straight line.

2. To place a straight line equal to a given straight line with one end at a given point.

3. To cut off from the greater of two given unequal straight lines a straight line equal to the less.

4.If two triangles have two sides equal to two sides respectively, and have the angles contained by the equal straight lines equal, then they also have the base equal to the base, the triangle equals the triangle, and the remaining angles equal to the remaining angles respectively, namely those opposite the equal sides.

5 . In isosceles triangles, the angles at the base equal one another, and, if the equal straight lines are produced further, then the angles under the base equal one another.

6. If in a triangle two angles equal one another, then the sides opposite to the equal angles also equal one another.

7.Given two straight lines constructed from the ends of a straight line and meeting in a point, there cannot be constructed from the ends of the same straight line, and on the side of it, two other straight lines meeting in another point and equal to the former two respectively, namely each equal to that from the same end.

8. If two straight lines have the two sides equal, to two sides respectively, and also have the base equal to the base, then they also have the anglers equal which are contained by the equal straight lines.

9. To bisect a given rectilinear angle.

10. To bisect a given finite straight line.

11. To draw a straight line at right angles to a given straight line from a given point on it.

12. To draw a straight line perpendicular to a given finite straight line from a given point not on it.

13. If a straight line stands on a straight line, then it makes either two right angles or angles whose sum equal to two right angles.
Vol.2 No 4 (Apr. 2009)

ISSN: 0974- 6846

14. If with any straight line and at a point on it, two straight lines not lying on the same side make the sum of the adjacent angles equal to two right angles, then the two straight lines are in a straight line with one another. 15. If two straight lines cut one another, then they make the vertical angles equal to one another.

Corollary: If two straight lines cut to one another then they will make the angles at the point of section equal to four right angles.

16. In any triangle, if one of the side is produced, the exterior angle is greater than either of the interior and opposite angles.

17. In any triangle, the sum of any two angles is less than two right angles.

18. In any triangle the angle opposite to the greater side is greater.

19. In any triangle, the side opposite the greater angle is greater.

20. In any triangle the sum of two sides is greater than the remaining one.

21. If from the ends of one of the sides of a triangle two straight lines are constructed meeting within the angle, then the sum of the straight lines so constructed is less than the sum of the remaining two sides of the triangle, but the constructed straight lines contain a greater angle than the angle contained by the remaining two sides.

22. To construct a triangle out of three straight lines which equal three given straight lines: thus it is is necessary that the sum of any two of the straight lines should be greater than the remaining one.

23. To construct a rectilinear angle equal to a given rectilinear angle on a given straight line and at a point on it.

24. If two triangles have two sides equal to two sides respectively, but have one of the angles contained by the equal straight lines greater than the other, then they also have the base greater than the base.

25. If two triangles have two sides equal to two sides respectively, but have the base greater than the base, then they also have the one of the angles contained by the equal straight lines greater than the other.

26. If two triangles have two angles equal to two angles respectively, and one side equal to one side, namely either the side adjoining the equal angles, or the opposite, then the remaining sides equal the remaining sides and the remaining angle equals the remaining angle.

27.If a straight line falling on two straight lines makes the alternate angles equal to one another, then the straight lines are parallel to one another.

28. If a straight line falling on two straight lines makes the exterior angle equal to the interior and opposite angle on the same side, or the sum of the interior angles on the same side equal to two right angles, then the straight lines are parallel to one another.

For easy follow up, here is a method:

Book I: basic plane geometry 
Def.I.23definition of parallel lines, one of many definitions in Bookl

Post.I.5: the parallel postulate

Common Notions: the axioms for magnitudes.

Prop.l.1: the first proposition which shows how to construct an equilateral triangle.

The congruence theorems for triangles: Prop.I.4,SAS, Propl.8;SSS, and Prop.I.26,ASA.

Propositions on isoscelestriangles.Prop.I.5, equal angles imply equal sides and the converse, Prop.I.6,equal sides imply equal angles.

Prop.I.9 and Prop.I.10 constructions to bisect angles and line segments.

Prop.I11 and Prop.I12 constructions to draw perpendicular lines

Prop.I.16,an exterior angle of a triangle is greater than either of the opposite interior anglesProp.I.20,the triangle inequality (the sum of two sides is greater than the third)

Prop.I.22, to construct a triangle with given sides

In Elements I, Euclid did NOT assume his fifth postulate to prove the above 28 propositions. To prove that the sum of the interior angles of a triangle is a straight angle, Euclid had to assume the fifth postulate which is also called "parallel postulate". After Euclid, almost all the famous mathematician attempted to deduce Euclid $\mathrm{V}$ from Euclid I -IV. But unfortunately, nobody was successful. The investigations devoted to the parallel postulate gave birth to its equivalent following propositions:

Propositions equivalent to Euclid V:

1. Through a point not on a given line there passes not more than one parallel to the line.

2. Two lines that are parallel to the same line are parallel to each other.

3. A line that meets one of two parallels also meets the other.

4. If two parallels are cut by a transversal, the alternate interior angles are equal.

5. There exists a triangle whose angle sum is a straight angle.

6. Parallel lines are equidistant from one another.

7. There exist two parallel lines whose distance apart never exceeds some finite value.

8. Similar triangles exist which are not congruent.

9. Any three points are collinear

10. Any three points are co-cyclic.

11. Through any point within any angle a line can be drawn which meets both sides of the angles.

12. There exists a quadrilateral whose angle sum is two straight angles.

13. Any two parallel lines can have a common perpendicular.

14, There exist a pair of straight lines everywhere equidistant from one another.

15. Two straight lines that intersect one another cannot be parallel to the third line.

16. There is no upper limit to the area of a triangle.
17. The sum of the angles is the same for every triangle.

18. There exists a quadrilateral of which all angles are right angles.

19. Phythacorean theorem.

20. There exists a pair of straight lines that are at constant distance from each other.

21. Given two parallel lines, any line that intersects one of them, also intersects the other.

22. If there is an acute angle such that a perpendicular drawn at every point on side will meet the other side also.

\section{Attempts to prove EuclidV}

For two thousand years, many attempts were made to prove the parallel postulate using Euclid's first four postulates. The main reason that such a proof was so highly sought after was that the fifth postulate isn't selfevident unlike the other postulates. If the order the postulates were listed in the Elements is significant, it indicates that Euclid included this postulate only when he realized he could not prove it or proceed without it.

Ibn al-Haytham (Alhazen) (965-1039), an Iraqi mathematician, made the first attempt at proving the parallel postulate using a proof by contradiction, where he introduced the concept of motion and transformation into geometry (David \& Hersh,1981). He formulated the Lambert quadrilateral, which Boris Abramovich Rozenfeld names the "Ibn al-Haytham-Lambert quadrilateral", and his attempted proof also shows similarities to Playfair's axiom.

Omar Khayyám (1050-1123) made the first attempt at formulating a non-Euclidean postulate as an alternative to the parallel postulate (Dunham,1991), and he was the first to consider the cases of elliptical geometry and hyperbolic geometry, though he excluded the latter. The Khayyam-Saccheri quadrilateral was also first considered by Omar Khayyam in the late 11th century in Book I of Explanations of the Difficulties in the Postulates of Euclid (Davis, 1999). Unlike many commentators on Euclid before and after him (including Giovanni Girolamo Saccheri), Khayyam was not trying to prove the parallel postulate as such but to derive it from an equivalent postulate: "Two convergent straight lines intersect and it is impossible for two convergent straight lines to diverge in the direction in which they converge." He recognized that three possibilities arose from omitting Euclid's Fifth; if two perpendiculars to one line cross another line, judicious choice of the last can make the internal angles where it meets the two perpendiculars equal (it is then parallel to the first line). If those equal internal angles are right angles, we get Euclid's Fifth; otherwise, they must be either acute or obtuse. He persuaded himself that the acute and obtuse cases lead to contradiction, but had made a tacit assumption equivalent to the fifth to get there.

Nasir al-Din al-Tusi (1201-1274), in his Al-risala alshafiya'an al-shakk fi'l-khutut al-mutawaziya (Discussion Which Removes Doubt about Parallel Lines) (1250), wrote detailed critiques of the parallel postulate and on 
Khayyám's attempted proof a century earlier. Nasir al-Din attempted to derive a proof by contradiction of the parallel postulate (Eves,1983). He was also one of the first to consider the cases of elliptical geometry and hyperbolic geometry, though he ruled out both of them.

Nasir al-Din's son, Sadr al-Din (sometimes known as "Pseudo-Tusi"), wrote a book on the subject in 1298, based on Nasir al-Din's later thoughts, which presented one of the earliest arguments for a non-Euclidean hypothesis equivalent to the parallel postulate. "He essentially revised both the Euclidean system of axioms and postulates and the proofs of many propositions from the Elements (Eves,1983; Hilbert,1999). His work was published in Rome in 1594 and was studied by European geometers. This work marked the starting point for Saccheri's work on the subject. Giordano Vitale (1633$1711)$, in his book Euclide restituo $(1680,1686)$, used the Khayyam-Saccheri quadrilateral to prove that if three points are equidistant on the base $A B$ and the summit $C D$, then $A B$ and $C D$ are everywhere equidistant.

Girolamo Saccheri (1667-1733) pursued the same line of reasoning more thoroughly, correctly obtaining absurdity from the obtuse case (proceeding, like Euclid, from the implicit assumption that lines can be extended indefinitely and have infinite length), but failing to debunk the acute case (although he managed to wrongly persuade himself that he had). Where Khayyám and Saccheri had attempted to prove Euclid's fifth by disproving the only possible alternatives, the nineteenth century finally saw mathematicians exploring those alternatives and discovering the logically consistent geometries which result.

In 1829, Nikolai Ivanovich Lobachevsky published an account of acute geometry in an obscure Russian journal, Kazan Messenger. This was a local university publication. Lobachevky'attempts to raise a wider audience had failed when his paper was rejected by Ostrogradski. He published "Geometrical investigations on the theory of parallels ' in 1840 which gives the clearest account of his work. The French publication of this work in Grelle's journal in 1837 brought his work on non-Euclidean geometry to a wide audience but the mathematical community was not ready to accept ideas so revolutionary. According to Lobachevsky, "All straight lines which in a plane go out from a point can, with reference to a given straight line in the same plane ,be divided into two classes-into cutting and non-cutting. The boundary lines o the $f$ one and the other class of those lines will be called parallel to the given line. Hence Lobachevsky had replaced the fifth postulate of Euclid by: There exist two lines parallel to a given line through a given point not on the line.

In 1831, János Bolyai included, in a book by his father, an appendix describing acute geometry, which, doubtlessly, he had developed independently of Lobachevsky.
Vol.2 No 4 (Apr. 2009)

ISSN: 0974- 6846
Carl Friedrich Gauss had actually studied the problem before that, but he didn't have the courage to publish any of his results. Because, he was the first person to really come to understand the problem of the parallels. He began work on the fifth postulate in 1792 while only 15 years old. By 1813 he made little progress and wrote: "In the theory of parallels we are even now not further than Euclid. This is a shameful part of mathematics. However by 1817 , Gauss had become convinced that the fifth postulate was independent of the other four postulates. He began to work out the consequences of a geometry in which more than one line can be drawn through a given point parallel to a given line. Perhaps most surprisingly of all Gauss never published this work but kept it a secret. At that time thinking was dominated by Kant who had stated that Euclidean geometry is the inevitable necessary of thought and Gauss disliked controversy. But Gauss discussed the theory of parallels with his friend, the mathematician Farkas Bolyai. And it was Gauss who first used the term "Non-Euclidean geometry". And it was Gauss who introduced the idea of 'surface curvature' on the basis of which Riemann later developed "Differential Geometry" that served as a foundation for Einstein's general theory of relativity.

Riemann gave an inaugural lecture on 10 June, 1854 in which he reformulated the whole concept of geometry which he saw as a space with enough extra structure to be able to measure things like length. This lecture was not published until 1868 Riemann briefly discussed a spherical geometry in which every line through a point $P$ not on a line $A B$ meets the line $A B$. In this geometry no parallels are possible. He wrote: "ust distinguish between unboundedness and infinite ...The unboundedness of space posses...a greater empirical certainty than any external experience. But its infinite extent by no means follows from this. Circles can be extended indefinitely since they have no ends. However, circles are of finite extent. Implicit in the first postulate; a straight line may be drawn between any two points- was the assumption that such a line is unique". So, Riemann modified Euclid's postulates 1,2 and 5 to as follows:

(1) Two distinct points determine at least one straight line.

(2) A straight line is boundless

(3) Any two straight lines in a plane intersect.

It is very easy to envisage the objects such as points, lines, and the plane of a geometry that satisfies the five modified postulates. Plane is a sphere, lines are the great circles, i.e circles whose plane passes through the center of the sphere points are regular points (but on the sphere of course). In this geometry, angle in a triangle sum up to more than a straight angle. The assumption of a single parallel line is equivalent to the fact that angles in a triangle sum to a straight angle. Furthermore, if this is true of a single triangle, this is also true of all possible triangles. In the geometry of Gauss, Lobachevsky and 
bolyai parallels are not unique. This is equivalent to the sum of the angles in a triangle being less than a straight angle. And again, this condition holds for all triangles provided it is true for any one of them. Riemann's spherical geometry completes a triad: no parallels and tha angle sum is always more than a straight angle.

The resulting geometries were later developed by Lobachevsky, Riemann and Poincaré into hyperbolic geometry (the acute case) and spherical geometry (the obtuse case).

The main thing is that Bolyai-Lobachevsky description of the new geometry had been proved not to be consistent. The first person to put the BolyaiLobachevsky non-Euclidean geometry on the same footing as the Euclidean geometry was Euginio Beltrami (1835-1900). In 1868 he write a paper, "Essay on the interpretation of non-Euclidean geometry" which produced a model for 2-dimensional non-Euclidean geometry within 3-dimensional Euclidean geometry. The model was obtained on the surface of revolution of a tractrix about its asymptote. But Beltrami's model was incomplete but it certainly gave a final decision on the fifth postulate of Euclid sine the model provided a settings in which Euclid's first four postulates held but the fifth did not hold. It reduced the problem of consistency of the axioms of non-Euclidean geometry to that of the consistency of the axioms of Euclidean geometry. Fortunately enough, Beltrami's work on a model of Bolyai-lobachevsky's non-Euclidean geometry was completed by Klein in 1871. Klein went further than this and gave models of other non-Euclidean geometries such as Riemann's spherical geometry .Klein's work based on a notion of distance defined by the Cayley in 1859 when he proposed a generalized definition for distance. Klein showed that there are three basically different types of geometry. In the Bolyai-Lobachevsky type of geometry, straight lines have two infinitely distant points. In the Riemannian type of spherical geometry, lines have no[or more precisely two imaginary] infinitely distant points. Euclidean geometry is a limiting case between the two where for each line there are coincident infinitely distant points.

The independence of the parallel postulate from Euclid's other axioms was finally demonstrated by Beltrami in 1868.

In a nutshell, Possidonius, proclus, Playfair, Nassiardin, John wallis, Legendre, Lambert, Wolfgang, Saccheri, Bolyai, Riemann, Hilbert, Peano, Veblen, Beltrami, Klein, Pascal, Monge, Mobius and many leading mathematicians worked on this problematic problem. People like Legendre, Saccheri and Gauss slept on this problem more than 50 long years in their

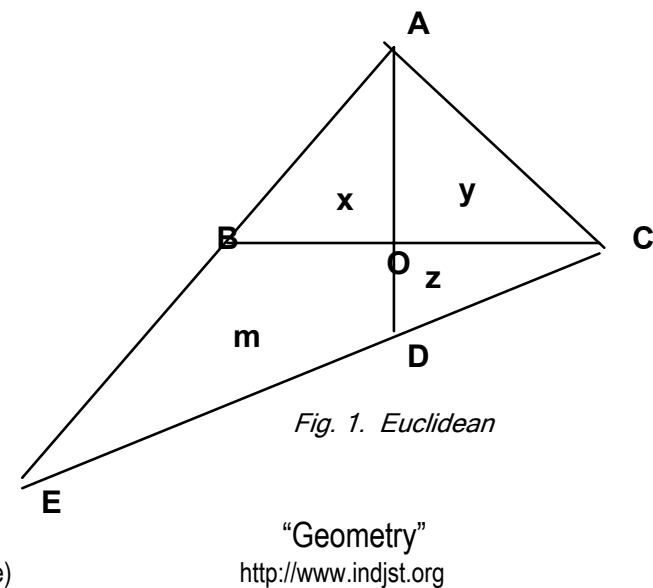

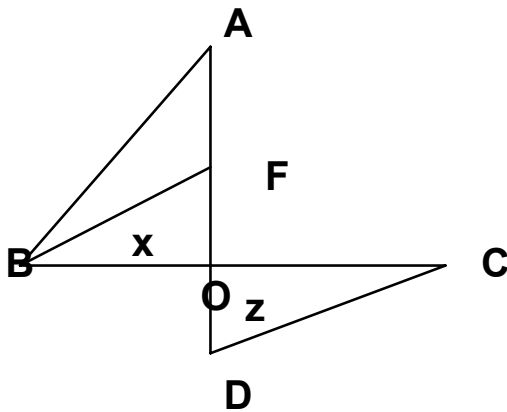

Fig. 2. Euclidean life. One side of the coin is that those 50 years of their precious youth. The famous Gauss found the famous formula for the sum of natural numbers[s $=\mathrm{n}(\mathrm{n}+1) / 2]$ when he was studying only at third standard. Such a great genius was not successful in his efforts to locate a solution for this problem. The author has spent 25 years on postulate geometry and found the following two results:

Geometrical Result: (Vide Fig. A)

$N A^{\prime}, N B^{\prime}, N C^{\prime}$ are the segments of a spheres whose north pole is $\mathrm{N}$. Choose a ponit $\mathrm{A}$ on

NA'. With center N, radius NA, describe an arc cutting AB' at $B$ and $A C^{\prime}$ at $C$.

So, $N A=N B=N C$

Take a point $\mathrm{F}$ on NA. With center $\mathrm{N}$, radius NF draw an arc meeting NB at $E$

And NC at D. So, NF = NE = ND

From (a) in triangle NAC, angle $\mathrm{NAB}=$ angle $\mathrm{NCB}$

And in triangle, NBC, angle NBC = angle NCB

From (c) and (d) we obtain that angles $\mathrm{NAB}=\mathrm{NBC}=\mathrm{NCB}=$ NBA $=90$ degree

Similarly from (b) we can show that angles, NFE $=$ NEF= $\mathrm{NED}=\mathrm{NDE}=90$ degree

From (e) and (f) we get that the sum of the interior angles of spherical quadrilateral

$B C D E$ is equal to 360 degrees

Algebraic Result

As long as algebra and geometry have been separated, their progress have been slow and their uses limited; but when these two sciences have been united, they have lent each mutual forces, and have marched together towards perfection."-- Joseph-Louis Lagrange.

\section{Construction}

In the Euclidean construction as shown in Fig. 1. $x, y, z$ and $m$ denote the sum of the interior angles of triangles $\mathrm{AOB}, \mathrm{AOC}, \mathrm{DOC}$ and quadrilateral $\mathrm{DOBE}$ respectively. Also let $a, b, c$ and $d$ respectively refer to the sum of the 
interior angles in triangles $A B C, A D C, B E C$ and $A E D$.

Sides $O B$ and $O C$ are equal

Side OA is greater than side OD

The angles AOD and $\mathrm{BOC}$ are straight angles and so their measures are equal to 180 degrees.Let $v$ be the value of this 180 degree

Using (3), $x+y=v+a$

$$
\begin{aligned}
& y+z=v+b \\
& z+m=2 v+c \\
& m+x=2 v+d
\end{aligned}
$$

(5) - (6) gives, $m+b=y+v+c$ Squaring (8), $m^{2}+a^{2}+2 m a=y^{2}+v^{2}+d^{2}+2 y v+2 y d+2 v d$ Squaring (9), $\mathrm{m}^{2}+\mathrm{b}^{2}+2 \mathrm{mb}=\mathrm{y}^{2}+\mathrm{v}^{2}+\mathrm{c}^{2}+2 y v+2 y c+2 v c$ $(4)+(6)=(5)+(7)=a+c=b+d$ Squaring (7), $a^{2}+c^{2}+2 c a=b^{2}+d^{2}+2 b d$ (8a) - (9a) given, $a^{2}-b^{2}+2 m a-2 m b=d^{2}-c^{2}+2 v(d-$ c) $+2 y(d-c)$

$a^{2}-b^{2}+2 m a-2 m b=d^{2}-c^{2}+2(d-c)[y+v]$

Putting (9) in RHS, $a^{2}-b^{2}+2 m a-2 m b=d^{2}-c^{2}+2(d-c)[m+$ $\mathrm{b}-\mathrm{c}]$

i.e $a^{2}-d^{2}+m[2 a-2 b-2 d+2 c]-b[b+2 c-2 d]+c[$ $2 d+c-2 c]=0$

Applying (10) in the second factor of LHS, $(a+d)(a-d$ )$-b[b+2 c-2 d]+c[2 d-c]=0$

From (10), $a-d=b-c$ and $b-a=c-d$

Putting these [i.e replacing a-d by b-c and c-d by $b-a$ ] in the above equation, $b[2 a-3 b+a+d]+c[2 d-c-a-d$ ] $=0$

Applying (10) in the second factor, $b[2 a-3 b+a+d]-$ $\mathrm{bc}=0$

i.e $b[3 a-3 b+d-c]=0$

From (10) we get $d-c=a-b$. Applying this $b[4 a-4 b]=0$

i.e $\quad a=b$

Analysing (4), (5) and (11) we have $z=x$

By construction sides $\mathrm{OB}$ and $\mathrm{OC}$ are equal

and side $O D$ is greater than side $O A$

Now look at Fig. 2. On OA, cut off $F$ such that $O D=O F$

By SAS correspondence, triangles $F O B$ and $C O D$ are congruent. But from (12) the sum of the interior angles of triangles $\mathrm{AOB}$ and $\mathrm{COD}$ are equal.

From this we obtain that the sum of the interior angles of triangles $A B O$ and $A B F$ are equal. Consequently, we get that the sum of the interior angles of triangle $A B F$ is equal to two right angles

\section{Discussion}

Since we have derived (g) and (13), without assuming Euclid's fifth postulate, (g) \& (13) establishes the parallel postulate. Fig. 1 can be extended to both hyperbolic and elliptic spaces. So, (13) will hold even in non- Euclidean spaces. But the mere existence of consistent models of non-Euclidean geometries demonstrates that Euclid $\mathrm{V}$ cannot be deduced from Euclid I to IV. But our result can NOT be challenged. Questioning (13) will force us to doubt the fundamental operations of number theory and algebra. In this study, application of classical algebra explored a masterpiece result in geometry. There is something hidden treasure of mathematics. Euclidean geometry is used in classical mechanics. Lobachevskian geometry helps to study the properties of objects/particles in quantum physics and Riemannian geometry is widely applied in general theory of relativity. A new field of geometry always gave rise to a new branch of physics. Present day physics is facing so many odds. There are many burning problems in physics, such as quantum gravity, tachyons missing mass, magnetic monopoles, anti-matter, blackholes, shadow of matter, antigravity and the origin of Universe and so on. Further probes will unlock the hidden mystery and may solve these problems.

\section{References}

1. Courant and Robbins (1941) What is mathematics? Oxord University Press.

2. Coxter HSM (1961) Introduction to geometry, John Wiley and Sons.

3. Coxter HSM (1988) Non-Euclidean geometry, MAA.

4. David FJ and Hersh (1981) The mathematical Experience, Houghton Mifflin Co.

5. Davis DM (1999) The nature and power of mathematics, Princeton University Press.

6. Devlin K (1977) Mathematics: The science of patterns, Scientific American Library.

7. Dunham W (1991) Journey through genius, Penguin books.

8. Effimov, NV (1972) Higher Geometry, Mir Publishers, Moscow

9. Eves H (1983) Great moments in mathematics after 1650, MAA.

10. Eves $H$ (1976) An introduction to the history of mathematics,. Holt, Rinechart and Winston, NY.

11. Heath, Sir Thomas L (1956) Euclid: The thirteen books of Elements, Dover Publications.

12. Hilbert D (1999) Foundations of geometry, Open Court.

13. Kac H and Ulam S (1968) Mathematics and Logic, Dover Publications.

14. Klein $F$ (2004) Elementary mathematics from an advanced standpoint: Geometry, Dover Publications.

15. Roberts S (1006) King of infinity space, Walker and Company.

16. Schwartzmann S (1994) The words of mathematics, MAA.

17. Sivasubramanian M (2009) A phenomenon in geometric analysis. Indian J.Sci. Technol. 2 (4), x-x.

18. Smilga (1972) In the search for the beauty, Mir Publishers,Moscow.

19. Swetz FJ (1996) From five fingers to infinity, Open Court.

20. Trudeau, Richard J (1987)The Non-Euclidean Revolution, Birkhauser, Boston.

21. Varadarajan VS (1998) Algebra in ancient and modern times, Hindustan Book Agency.

22. Wikipedia [Parallel Postulate] Section2,Proposition 7. 